\begin{tabular}{|c|r|r|r|r|r|}
\hline Revista Duazary & ISSN: $1794-5992$ & Vol. 14 & No. 2 & $197-203$ & Julio - Diciembre de 2017 \\
\hline \multicolumn{6}{|c|}{ DOI: http://dx.doi.org/10.21676/2389783X.1965 } \\
\hline
\end{tabular}

\title{
EVALUACIÓN CLÍNICA DE LA INCONTINENCIA URINARIA EN LA CONSULTA GINECOLÓGICA
}

\section{CLINICAL EVALUATION OF URINARY INCONTINENCE IN GYNECOLOGIC CONSULTATION}

TITULO CORTO: EVALUACIÓN CLÍNICA DE LA INCONTINENCIA URINARIA

Franklin J. Espitia-de la Hoz ${ }^{1}$

Recibido en abril 20 de 2016

Aceptado en diciembre 06 de 2016

Publicado en línea en mayo 15 de 2017

\section{Resumen}

Se objetivó revisar la literatura existente respecto a la valoración clínica de la incontinencia urinaria femenina en la consulta ginecológica. Se hizo una exploración de la literatura médica en bases de datos electrónicas, libros de textos impresos, revistas indexadas y sociedades científicas reconocidas a fin de identificar la literatura relevante en la valoración clínica del escape de orina en la consulta ginecológica. En la actualidad, la evidencia sobre este tema no permite sugerir un examen clínico específico para valorar la incontinencia en una consulta ginecológica, pero la correlación de hallazgos entre uno y otro facilita el mismo. La literatura muestra que los métodos de evaluación clínica de la incontinencia urinaria en la consulta ginecológica juegan un importante papel en el adecuado tratamiento de la misma y en el correcto diagnóstico de las pacientes; Por lo tanto, se hace necesario volver los ojos a la adecuada evaluación clínica.

Palabras clave: Incontinencia; diagnóstico; síntomas; urgencia; uretra.

\section{Abstract}

It is aimed to review the literature regarding the clinical evaluation of female urinary incontinence in gynecologic consultation. An exploration of the literature in several different electronic databases, books printed texts in indexed journals and recognized scientific societies, to identify relevant literature was trying to find the best evidence in the clinical assessment of urine leakage in gynecological consultation. They are not allowing sufficient scientific documents suggest which is the most specific clinical examination in the assessment of incontinence in gynecologic consultation, but the correlation of findings from each other, facilitates the same. The literature shows that the methods of clinical evaluation of urinary incontinence in gynecologic consultation play an important role in the correct diagnosis of patients and proper treatment thereof; therefore, it is necessary to turn our eyes to the appropriate clinical evaluation in gynecological consultation.

Keywords: Incontinence; diagnostic; symptoms; urgency; urethra. 


\section{INTRODUCCIÓN}

$\mathrm{L}$ a incontinencia urinaria tiene diferentes definiciones, pero la tendencia actual es tratar de homogeneizarlas y concluir en un solo concepto, que es el propuesto por la Sociedad Internacional de Continencia (ICS) el cual la define como la presencia de cualquier escape involuntario de orina ${ }^{1,2}$ que supone un problema social, de carácter relevante debido a su prevalencia, connotaciones psicosociales y económicas ${ }^{3}$.

Según la sintomatología, se distinguen tres tipos de incontinencia clasificados por la ICS:

a) Incontinencia urinaria de esfuerzo: es el escape involuntario de orina al realizar maniobras de Valsalva que aumenten la presión intraabdominal como estornudar, hacer movimientos bruscos, toser e, incluso, reírse $^{4,5,2}$; puede ser ocasionada por debilidad del piso pélvico o presencia de daño a nivel uretral, y puede estar asociada a múltiples factores de riesgo o presencia de daño en el esfínter uretral, aunado a diversos factores de riesgo ${ }^{6}$.

b)Incontinencia urinaria de urgencia: es el escape involuntario de orina que se asocia a un fuerte deseo de orinar, conocido como «urgencia miccional», debido a contracciones involuntarias del musculo detrusor?

c) Incontinencia urinaria mixta: es la sensación de escape involuntario de orina, que se vincula tanto a la urgencia como al esfuerzo ${ }^{8}$.

La incontinencia urinaria, según la base del origen de la pérdida de orina, puede darse por vía extrauretral e intrauretral. La extrauretral, consiste en el escape de orina a través de un orificio anormal entre el tracto urinario y el exterior (fístulas, uréter ectópico, divertículo uretral, etc.); y la intrauretral, se refiere a las incontinencias que ocurren durante circunstancias que incrementan la presión intraabdominal (ejercicio, estornudo, tos, etc.) ${ }^{9,10}$.

A la incontinencia urinaria de esfuerzo también se le clasifica en ${ }^{11,12}$ :

198

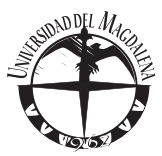

a) Tipo 0: existen síntomas de incontinencia urinaria, pero no se revela en el examen físico ni en la urodinamia. La base vesical se mantiene por encima del pubis, no desciende con la maniobra de Valsalva.

b) Tipo I: existen síntomas de incontinencia urinaria, se revela al examen físico y en la urodinamia. La base vesical se mantiene por encima del pubis tanto en reposo como durante la maniobra de Valsalva, pero con deslizamiento y abertura de la uretra, asociada a fuga de orina. Se evidencia leve hipermovilidad uretral.

c) Tipo IIa: se presentan síntomas de incontinencia urinaria que se revelan al examen físico y en la urodinamia. La base vesical se mantiene por encima del pubis en quietud, pero se desplaza con la maniobra de Valsalva por debajo del pubis, asociada a fuga de orina. La hipermovilidad uretral se revela con el esfuerzo.

d) Tipo IIb: se presentan síntomas de incontinencia urinaria que se revelan al examen físico y en la urodinamia. La base vesical permanece por debajo del pubis en quietud, y se desplaza más durante la maniobra de Valsalva, asociada a fuga de orina. Se evidencia la anómala ubicación de la unión vesicouretral desde la quietud. La hipermovilidad uretral se revela con el esfuerzo.

e) Tipo III: se presentan síntomas de incontinencia urinaria constante, tanto diurna como nocturna, con ínfimo esfuerzo. La uretra habitúa estar fija y no revela movilidad uretral importante, el cuello vesical suele estar abierto en reposo, con fuga continua de orina.

\section{MATERIALES Y MÉTODOS}

Se realizaron búsquedas con las palabras clave: incontinencia; diagnóstico; síntomas; urgencia; uretra. La exploración bibliográfica se ejecutó en las bases de datos electrónicas EBSCO, Elsevier, Interscience, Medline, Ovid, Pubmed, SciELO, Scopus, (1980 al 2015), Cochrane Central Register of Controlled Trials (enero 01 de 2005 a mayo 31 del 2015), así como en textos impresos, revistas indexadas y sociedades médicas reconocidas, tales como: International Continence Society, International Academy of Pelvic Surgery e International Urogynecological Association; tratando de encontrar la mejor evidencia en la evaluación clínica de la incontinencia urinaria en la consulta ginecológica. Se analizaron artículos en inglés y en español.

\section{RESULTADOS}

El rastreo en la base de datos electrónicas mostró 105 títulos, de los que se excluyeron 40 debido a que no respondían la pregunta planteada en nuestro estudio. Finalmente, se seleccionaron 65 artículos divididos de la siguiente manera: 47 revisiones, 15 estudios de casos y controles, y tres estudios de corte transversal. 


\section{Epidemiologia}

La incontinencia urinaria predomina en la estadística de los adultos y es dos a cuatro veces más frecuente en mujeres que en hombres. La incidencia crece de forma lineal con la edad ${ }^{13,14}$ : en la gente joven se mantiene entre el $20 \%$ y $30 \%$, aumentando en la adultez entre el $30 \%$ y $40 \%$, mientras que en el grupo de más edad presenta un aumento persistente de 30 a $50 \%{ }^{15,16}$. Si bien la incontinencia urinaria de esfuerzo muestra su mayor prevalencia en las mujeres de 40 a 54 años de edad ${ }^{17,18}$, la prevalencia de la incontinencia urinaria femenina puede variar entre el $45 \%{ }^{19}$ y el $69 \%{ }^{20}$ de acuerdo a la población de estudio y a los criterios diagnósticos.

La incontinencia urinaria se hace presente durante el embarazo en entre el $36 \%$ y el $58 \%$ de los casos, y se soluciona en la mayoría de los mismos sin intervenciones ${ }^{21,22}$. En el puerperio, por su parte, varía entre el $27 \%$ y el $33 \%^{23,24}$.

En todos los grupos etarios la incontinencia urinaria de esfuerzo es el patrón más usual, presentándose en cerca de la mitad de las mujeres con incontinencia (50\%); le continua en frecuencia la incontinencia mixta (32\%), seguida de la incontinencia de urgencia pura, que es la menos frecuente $(14 \%)$ ) y siendo a su vez más frecuente la incontinencia urinaria en mujeres de raza blanca que en mujeres de raza negra ${ }^{26}$.

\section{Factores de riesgo}

La incontinencia urinaria es una disfunción de origen multifactorial por lo que, en esta colectividad, su etiología es mal definida ${ }^{27}$. Existen factores de riesgo establecidos como la obesidad, que aumenta tres veces más el riesgo de incontinencia urinaria, así como la paridad, el antecedente familiar, los síntomas urinarios y las actividades de alto impacto. Además, se suelen asociar a su desarrollo factores como antecedentes de radiación pélvica, deterioro funcional y/o cognitivo, embarazo, envejecimiento, estreñimiento, tabaquismo, infecciones del tracto urinario, alteraciones del sistema nervioso central, tos crónica y tumores pélvicos. Así también, existen factores sugeridos como los partos vaginales, la edad, la raza, el tabaquismo, el consumo de cafeína, la atrofia vaginal y la histerectomía ${ }^{28,29}$.

\section{Diagnóstico}

La historia clínica exhaustiva y la exploración física (el examen físico general, exploración abdominal focalizada a nivel del hipogastrio, valoración de la sensibilidad perianal y de los reflejos lumbosacros y exploración ginecológica) hacen parte del arsenal semiológico obligatorio para realizar el diagnóstico de la incontinencia urinaria ${ }^{30,31}$.

El cuestionario de las tres preguntas de la incontinencia (3IQ) ha sido desarrollado para categorizar la incontinencia y guiar el plan de tratamiento inicial. En la incontinencia de urgencia la sensibilidad es del $75 \%$, así que el $25 \%$ de las incontinentes no serán detectadas; mientras que la especificidad es del $77 \%$, por lo que el $23 \%$ de féminas con diferentes tipos de incontinencia serán incorrectamente abordadas por incontinencia de urgencia. En la incontinencia de esfuerzo, la sensibilidad es del $86 \%$, así que el $14 \%$ de las mujeres que la padecen no son manifiestas; y la especificidad es del $60 \%$, por lo que el $40 \%$ de las mujeres con otras incontinencias serán incorrectamente abordadas por incontinencia de esfuerzo $^{32}$. De ahí que la anamnesis deba ser la prioridad, y que deba acompañarse de pruebas adicionales o complementarias.

Una revisión que incluyó 15 estudios distintos de mujeres sintomáticas de patología urinaria, con la urodinamia como Gold estándar ${ }^{33,34}$, permitió encontrar sensibilidad en la historia clínica de 0,92 (IC $95 \%, 0.91-0.93$ ), con especificidad de 0,56 (IC 95\% 0.53-0.60), VPP 0,75 y VPN 0,77 ; lo que refuerza la utilidad diagnostica de la historia clínica aunque, por sí misma, no sea una pieza diagnóstica confiable ${ }^{35}$.

\section{Pruebas objetivas para estudio de incontinencia urinaria}

a) Prueba de esfuerzo o Test de Boney: se realiza con vejiga llena una prueba de esfuerzo (toser y tensión) tanto en bipedestación como en posición ginecológica, tratando de visualizar fugas de orina secundarias a incompetencia esfinteriana. El Test de Boney fue comparado en seis estudios con la urodinamia ${ }^{36,37}$ para el diagnóstico de incontinencia urinaria de esfuerzo, mostrando sensibilidad del 0.85 (IC $95 \%, 0,78-0,91$ ) y especificidad del 0.83 (IC 95\%, 0,74-0,90); lo que lo hace no despreciable en el arsenal diagnóstico de la práctica ginecológica ${ }^{38,39}$.

b)La prueba del hisopo o Q-TIP test: es un sencillo procedimiento usado para cuantificar la movilidad de la unión uretrovesical. Se introduce una torunda de algodón o hisopo, estéril y lubricado, a través de la uretra hasta el nivel en que se supera la presión del esfínter uretral interno (a una distancia de 3,5 a 
$4,5 \mathrm{~cm}$ del meato uretral). Posteriormente, se le solicita a la mujer que tosa y se observa el movimiento de la torunda o hisopo; este debe ser inferior a 30 grados si no presenta hipermovilidad uretral. Un cambio de ángulo inferior a $30^{\circ}$, ante evidencia de incontinencia, sugiere deficiencia esfinteriana intrínseca. Tiene utilidad diagnóstica en la incontinencia urinaria genuina de esfuerzo, si bien su positividad no es diagnóstica ${ }^{40,41}$. Infortunadamente el test caracteriza un defecto estructural en la pelvis, pero no funcional, ya que las mujeres pueden presentar hipermovilidad uretral sin incontinencia ${ }^{42}$.

c) Test de Marshall-Bonney: radica en revelar la fuga de orina con el Valsalva. Se solicita a la mujer que tosa fuerte, observándose el descenso de la unión uretrovesical. En el caso de existir incontinencia urinaria, se verá el escape de orina. Posteriormente, se eleva el cuello vesical con dos dedos, presionando a los lados, y se le solicita a la paciente que tosa de nuevo. Si no se demuestra fuga de orina, se considera positiva: sugiere incontinencia urinaria por hipermovilidad uretral ${ }^{43}$. Es de anotar que algunos autores desaconsejan su utilización para el diagnóstico de hipermovilidad uretral ${ }^{42}$.

d) PAD test o Prueba de la compresa (de una hora o de 24 horas): puede diagnosticar la incontinencia de forma precisa y objetivar su grado. A la mujer se le coloca una compresa que se pesa previamente y se vuelve a pesar luego de cumplido el tiempo establecido. La ganancia de peso orienta acerca de la magnitud de la incontinencia ${ }^{44,45}$ (continente: $\leq 1$ gramo, leve: 1,19,9 gramos, moderado $10-49,9$ gramos y severa $\geq 50$ gramos).

e) Diario miccional ${ }^{46}$ : es la anotación de las micciones en una hoja diseñada para tal fin. Se anotan las horas a las que se realiza la micción y los volúmenes orinados. Este diario es valioso porque cuantifica el número de evacuaciones (frecuencia diurna y nocturna), los eventos de urgencia e incontinencia; asimismo, aporta otras circunstancias como: toma de líquidos, disposición de aguantar hasta el límite, y valora también el número y tipo de pañales empleados como protección. A su vez, es significativo porque registra, en el día a día, las evacuaciones miccionales que suceden en las tareas diarias y en las situaciones ordinarias de la mujer. 200 Su sensibilidad es del $68 \%$, con una proporción de falsos negativos del $32 \%$ en el diagnóstico de cualquier fuga de orina al compararse con la urodinamia ${ }^{47}$. Si el examen es de 48 horas, su sensibilidad sube al $92 \%$ con especificidad del $72 \%$, con un $8 \%$ de falsos negativos y con un $28 \%$ de falsos positivos en el diagnóstico de incontinencia urinaria de esfuerzo ${ }^{48}$, justificando la trascendencia de la prueba.

Existen otros factores en el interrogatorio de la historia clínica que cobran importancia a la hora de esclarecer diagnósticos diferenciales de incontinencia como:

- La capacidad cognitiva de reconocer y reaccionar a la sensación de tener la vejiga llena.

- La movilidad y destreza manual para alcanzar servicio sanitario.

- Las infecciones del tracto urinario que causan la inflamación de la mucosa de la vejiga.

- El uso de medicamentos, causa importante de poliuria, retención y escape de la orina.

\section{Pruebas complementarias}

Se han diseñado cuestionarios estandarizados que pueden guiar el diagnóstico y la clasificación de la incontinencia, además de permitir valorar la respuesta al tratamiento y reconocer el impacto de los síntomas en la calidad de vida de la mujer con fuga de orina, como son: el Test de severidad de Sandvick ${ }^{49}$, el cuestionario ICIQ-SF (International Consultation on Incontinence Questionnaire Short-Form) $)^{50}$, el King's Health Questionnaire ${ }^{51}$, entre otros; por lo que sugiero que se le realice a todas las pacientes que consultan por incontinencia urinaria.

\section{Estudios de laboratorio}

Urianálisis $^{52}$ : describe un conjunto de exámenes de tamizaje útiles para revelar patología renal o del tracto urinario.

Función renal ${ }^{53}$ : junto con los síntomas referidos por la paciente, pueden ayudar a hacer un diagnóstico sindrómatico y una evaluación de los mismos.

Urocultivo $^{54}$ : en los casos de incontinencia de urgencia 0 mixta si se acompañan de manifestaciones irritativas u obstructivas del tracto urinario.

\section{DISCUSIÓN}

Existe evidencia de que los métodos de evaluación clínica de la incontinencia urinaria, en la consulta ginecológica, juegan un importante papel en su correcto diagnóstico ${ }^{4}$. Muchos autores discuten lo innecesario de la urodinamia 
en las mujeres incontinentes, considerándola un método diagnóstico costoso, imperfecto, invasivo, que consume tiempo y que no reproduce perfectamente los síntomas, además de no estar al alcance de todos ${ }^{55}$. Esto hace obligatorio volver los ojos a la adecuada evaluación clínica y semiológica en la consulta ginecológica. Sin embargo, aunque la urodinamia es un instrumento que no es de rutina, es de gran ayuda para el estudio del comportamiento de variables fisiológicas en el tracto urinario durante el acto de micción. Tiene indicaciones claras y precisas como: incontinencia urinaria sin comprobación clínica, trastornos neurológicos, recidiva luego de cirugía, falla en el tratamiento médico, predominio de síntomas irritativos, sospecha de lesión del esfínter, respaldo médico legal y corrección quirúrgica de prolapso.

Los métodos clínicos diagnósticos que determinan o facilitan la valoración de la fuga de orina en la mujer, se pueden utilizar con relativa facilidad en la consulta de ginecología ${ }^{35}$ ya que han sido desarrollados para ser usados con este propósito. Es así como considero que, dentro de la evaluación de la mujer incontinente, se debe realizar un exhaustivo interrogatorio y el más completo examen físico ${ }^{32}$, el cual amerita incluir todas las pruebas clínicas diagnósticas aquí descritas antes de pensar en ayudas diagnósticas innecesarias, invasivas o costosas ${ }^{40}$ independientemente de lo muy sofisticadas, cosmopolitas y avanzadas que parezcan.

\section{CONCLUSIÓN}

La incontinencia urinaria es un trastorno con una alta incidencia y prevalencia en el sexo femenino; por ello, es imprescindible el adecuado diagnóstico clínico de la misma, puesto que existe una relación excelente entre la correcta evaluación y los buenos resultados diagnósticoquirúrgicos.

Existe poca evidencia que permita sugerir una recomendación precisa y contundente de cuál es el examen clínico más específico para diagnosticar la fuga de orina en la mujer; no obstante, la correlación de hallazgos entre uno y otro facilita el mismo.

Un escaso apoyo en la base de la vejiga y el pobre sostén de la unión uretrovesical son las principales explicaciones de la incontinencia urinaria.

El Gold estándar para diagnosticar la fuga de orina con el esfuerzo sigue siendo la urodinamia, pero la historia clínica, la anamnesis y el examen físico en el inicio del estudio de la misma no son despreciables.

La evidencia actual demuestra que el correcto diagnóstico de una mujer con sintomatología incontinente se puede realizar utilizando métodos básicos, que están disponibles y al acceso en todos los consultorios de los profesionales de la salud.

\section{DECLARACIÓN SOBRE CONFLICTOS DE INTERESES}

Los autores no reportan conflictos a declarar relacionados con la investigación.

\section{REFERENCIAS BIBLIOGRÁFICAS}

1. Melville J, Katon W, Delaney K, Newton K. Urinary incontinence in US women: a population-Based Study. Arch Intern Med. 2005 Mar 14; 165 (5):537-42.

2. Abrams P, Cardozo L, Fall M, Griffiths D, Rosier P, Ulmsten $U$. The standardization of terminology of lower urinary tract function: report from the Standardization Subcommittee of the International Continence Society. Urology. 2003 Jan; 61(1):37-49.

3. Kegel AH. Progressive resistance exercise in the functional restoration of the perineal muscles. Am J Obstet Gynecol. 1948 Aug; 56(2):238-48.

4. Dass AK, Lo TS, Khanueng-kitkong S, Tan YL. Diagnosis and conservative management of female stress urinary incontinence. Gynecology and Minimally Invasive Therapy. 2013 may; 2(2):48-51.

5. Minassian V, Stewart W, Wood C. Urinary Incontinence in Women: Variation in Prevalence Estimates and Risk Factors. Obstetrics \& Gynecology. 2008 Feb; 111(2 Pt 1):324-31.

6. Meneses M, Pereira M, Hextall A. Predictors of female urinary incontinence at midlife and beyond. Maturitas. 2010 Feb; 65(2):167-71.

7. Chapple CR, Khullar V, Gabriel Z, Muston D, Bitoun CE, Weinstein D. The effects of antimuscarinic treatments in overactive bladder: an update of a systematic review and meta-analysis. Eur Urol. 2008 Sep; 54(3):543-62.

8. Hannestad YS, Rortveit G, Sandvik H, Hunskaar S. A community-based epidemiological survey of female urinary incontinence: The Norwegian EPINCONT study. Epidemiology of Incontinence in the County of NordTrondelag. J Clin Epidemiol. 2000 Nov; 53(11):1150-7.

9. McCord J, Ginecología Quirúrgica. Madrid: Editorial medica panamericana; 2008. 
10. Diokno AC, Brock BM, Brown MB, Herzog AR. Prevalence of urinary incontinence and other urological symptoms in the no ninstitutionalized elderly. J Urol. 1986 Nov; 136 (5):1022-5.

11. Blaivas JG, Olsson CA. Stress incontinence: classification and surgical approach. J Urol. 1988 Apr; 139(4):727-31.

12. McGuire EJ. Diagnosis and treatment of intrinsic sphincter deficiency. Int J Urol. 1995; 2(Suppl 1):7-10.

13. Wein AJ. Voiding function and dysfunction, bladder physiology and pharmacology, and female urology. J Urol. 2010 Nov; 184(5):2029-34.

14. Simeonova Z, Bengtsson C. Prevalence of urinary incontinence among women at a Swedish Primary Health Care Centre. Scand J Prim Health Care. 1990; 8:4, 203-6.

15. Carneiro KS. Prevalência e fatores de risco associados à Incontinência Urinária em mulheres acima de 15 anos, em uma comunidade, no Brasil. Estudo por abordagem direta. [tese]. São Paulo (SP): Escola Paulista de Medicina, Universidade Federal de São Paulo; 2006.

16. Pollard ME, Morrisroe S, Anger JT. Outcomes of pregnancy following surgery for stress urinary incontinence: a systematic review. J Urol. 2012 Jun; 187(6):1966-70.

17. Martínez C, Flores O, García de Alba J, Velázquez P, González M, Márquez R. Prevalencia de incontinencia urinaria y anal en mujeres de la zona metropolitana de Guadalajara. Ginecol Obstet Mex 2006; 74 (6): 300-5.

18. Fultz NH, Herzog AR. Epidemiology of urinary symptoms in the geriatric population. Urol Clin North Am. 1996 Feb; 23(1):1-10.

19. Melville JL, Katon W, Delaney K, Newton K. Urinary incontinence in US women. a population-based study. Arch Intern Med. 2005; 165(5):537-42.

20. Swithinbank LV, Donovan JL, du Heaume JC, Rogers CA, James MC, Yang Q, Abrams P, et al. Urinary symptoms and incontinence in women: relationships between occurrence, age, and perceived impact. Br J Gen Pract. 1999; 49(448):897-900.

21. Wesnes SL1, Rortveit G, Bo K, Hunskaar S. Urinary incontinence during pregnancy. Obstet Gynecol. 2007 Apr; 109(4):922-8.

22. Dolan LM, Walsh D, Hamilton S, Marshall K, Thompson $\mathrm{K}$, Ashe RG. A study of quality of life in primigravidae with urinary incontinence. Int Urogynecol J Pelvic Floor Dysfunct. 2004 May-Jun; 15(3):160-4.

202 23. Thom DH, Rortveit G. Prevalence of postpartum urinary incontinence: a systematic review. Acta Obstet Gynecol Scand. 2010 Dec; 89(12):1511-22.

24. Serati M, Salvatore S, Khullar V, Uccelia S, Bertelli E, Ghezzi F et al. Prospective study to assess risk factors for pelvic floor dysfunction after delivery. Acta Obstet Gynecol Scand. 2008; 87(3):313-8.
25. Haylen BT, de Ridder D, Freeman RM, Swift SE, Berghmans $B$, Lee $J$, et al. An International Urogynecological Association (IUGA)/International Continence Society (ICS) joint report on the terminology for female pelvic floor dysfunction. Neurourol Urogynecol J. 2010; 29(1):4-20.

26. Digesu GA, Athanasiou S, Chaliha C, Michalas S, Salvatoore S, Selvaggi L, et al. Urethral retro-resistance pressure and urodynamic diagnoses in women with lower urinary tract symptoms. BJOG. 2006; 113:34-8.

27. Bernstein T. The pelvic floor muscles: muscle thickness in healthy and urinary-incontinent women measured by perineal ultrasonogaphy with reference to the effect of pelvic floor training. Estrogen receptor studies. Neurol Urodyn. 1997; 16(4):237-75.

28. Fritel X, Ringa V, Varnoux N, Fauconnier A, Piault S, Bréart $\mathrm{G}$. Mode of delivery and severe stress incontinence. a cross-sectional study among 2,625 perimenopausal women. BJOG. 2005 Dec; 112(12):1646-51.

29. Grodstein F, Fretts R, Lifford K, Resnick N, Curhan G. Association of age, race, and obstetric history with urinary symptoms among women in the Nurses' Health Study. Am J Obstet Gynecol. 2003 Aug; 189(2):428-34.

30. Lucas MG1, Bosch RJ, Burkhard FC, Cruz F, Madden TB, Nambiar AK, Neisius A, et al. Guidelines in Urinary Incontinence, European Association of Urology 2012, www.uroweb.org. 2012 Dec; 62(6):1118-29.

31. Leach GE, Dmochowski RR, Appell RA, Blaivas JG. Female Stress Urinary Incontinence Clinical Guidelines Panel summary report on surgical management of female stress urinary incontinence. The American Urological Association. J Urol. 1997 Sep; 158(3 Pt 1):875-80.

32. Brown JS, Bradley CS, Subak LL, Richter HE, Kraus SR, Brubaker L, et al. The sensitivity and specificity of a simple test to distinguish between urge and stress urinary incontinence. Ann Intern Med. 2006 May 16; 144(10):715-23.

33. Sunshine, T. J., \& Glowacki, G. A. Clinical correlation of urodynamic testing in patients with urinary incontinence. Journal of Gynecologic Surgery, 1989; 5(1): 93-8.

34. Cundilf GW, Harris RL, Coates W, Bump RC. Clinical predictors of urinary incontinence in women. Am J Obstet Gynecol. 1997 Aug; 177(2):262-6.

35. Jensen JK, Nielsen FR, Ostergard DR. The Role of patient history in the diagnosis of urinary incontinence, diagnosis of urinary incontinence. Obstet Gynecol 1994; 83(5 Pt2):904-10.

36. Türker P, Kilic G, Tarcan T. The presence of transurethral cystometry catheter and type of stress test affect the measurement of abdominal leak point pressure (ALPP) in women with stress urinary incontinence (SUI). Neurourol Urodyn. 2010 Apr; 29(4):536-9. 
37. Scotti RJ, Myers DL. A comparison of the cough stress test and single channel cystometry with multichannel urodynamic evaluation in genuine stress incontinence. Obstet Gynecol. 1993 Mar; 81(3):430-3.

38. Resnick NM, Brandeis GH, Baumann MM, OuBeau CE, Yalla SV. Misdiagnosis of urinary incontinence in nursing home women: prevalence and a proposed solution. Neurourol Urodyn. 1996; 15(6):599-613.

39. Jorgensen L, Lose G, Andersen JT. One-hour padweighing test for objective assessment of female urinary incontinence. Obstet GynecoI. 1987 Jan; 69(1):39-42.

40. Crystle CD, Charme LS, Copeland WE. Q-tip test in stress urinary incontinence. Obstet Gynecol. 1971 Aug; 38(2):313-5.

41. Karram MM, Bhatia NN. The Q-tip test: standardization of the technique and its interpretation in women with urinary incontinence. Obstet Gynecol. 1988 Jun; 71(6 Pt 1):807-11.

42. Montz F, Stanton S. Q-tip test in female urinary incontinence. Obstet Gynecol. 1986 Feb; 67(2):258-60.

43. Miyazaki FS.The Bonney test- a reassessment. Am J. Obstet Gynecol. 1997 Dec; 177(6):1322-8.

44. Aslan E, Beji NK, Coskun A, Yalcin O. An assessment of the importance of pad testing in stress urinary incontinence and the effects of incontinence on the life quality of women. Int Urogynecol J Pelvic Floor Dysfunct. 2003 Nov; 14(5):316-9.

45. Franco AV, Lee F, Fynes MM. Is there an alternative to pad tests? Correlation of subjective variables of severity of urinary loss to the 1-h pad test in women with stress urinary incontinence. BJU Int. 2008 Aug; 102(5):586-90.

46. Ku JH, Jeong IG, Lim DJ, Byun SS, Paick JS, Oh SJ. Voiding diary for the evaluation of urinary incontinence and lower urinary tract symptoms: prospective assessment of patient compliance and burden. Neurourol Urodyn. 2004; 23(4):331-5.
47. Versi E, Cardozo L. The use of pad test in the investigation of female urinary incontinence. J. Obstet Gynecol. 1988; 8(12):270-3.

48. Versi E1, Orrego G, Hardy E, Seddon G, Smith P, Anand D. Evaluation of the home pad test in the investigation of female urinary incontinence. Br J. Obstet Gynecol. 1996 Feb; 103(2):162-7.

49. Sandvik H1, Seim A, Vanvik A, Hunskaar S. A severity index for epidemiological surveys of female urinary incontinence: comparison with 48-hour pad-weighing tests. Neurourol Urodyn. 2000; 19(2):137-45.

50. Espuña M, Rebollo P, Puig M. Validation of the Spanish version of the International Consultation on Incontinence Questionnaire-Short Form. A questionnaire for assessing the urinary Incontinence. Med Clin (Barc) 2004 Mar; 122(8):288-92.

51. Espuña-Pons M, Dilla T, Castro D, Carbonell C, Casariego J, Puig-Clota M. Analysis of the value of the ICIQ-UI SF questionnaire and stress test in the differential diagnosis of the type of urinary incontinence. Neurouroll Urodyn. 2007; 26(6):836-41.

52. Graff EL. Análisis de Orina, Atlas de Color. Buenos Aires: Editorial Médica Panamericana; 1987.

53. Hamburguer J, Richet G, Crosnier J, Funck Brentano, Ducrot H. Exploración funcional del riñón. Edi. Elicien, España, Trad. Martínez Sauret A; 1965.

54. Alós JI. Epidemiology and etiology of urinary tract infections in the community. Antimicrobial susceptibility of the main pathogens and clinical significance of resistance. Enferm Infecc Microbiol Clin. 2005; 23(4):3-8.

55. Nager CW, FitzGerald M, Kraus SR, Chai TC, Zyczynski $\mathrm{H}$, Sirls L, et al. Urodynamic measures do not predict stress continence outcomes after surgery for stress urinary incontinence in selected women. J Urol. 2008 Apr; 179(4):1470-4.

Para citar este artículo: Espitia-de la Hoz F. Evaluación clínica de la incontinencia urinaria en la consulta ginecológica. Duazary. 2017 julio; 14 (2): 197 - 203. Doi: http://dx.doi. org/10.21676/2389783X.1965 